\title{
Response of Nano-Reinforced Cementitious Composites Using Natural and Commercial Dispersants ${ }^{\dagger}$
}

\author{
Qareeb Ullah Anwari 1,*, Rao Arsalan Khushnood 1, Sajjad Ahmad 2, Shad Muhammad ${ }^{1}$ and \\ Jean-Marc Tulliani ${ }^{3}$ \\ 1 School of Civil and Environmental Engineering, National University of Sciences and Technology, \\ Islamabad 44000, Pakistan; arsalan.khushnood@nice.nust.edu.pk (R.A.K.); smuhammad@live.com (S.M.) \\ 2 Department of Structural, Geotechnical and Building Engineering, Politecnico di Torino, 10129 Turin, Italy; \\ sajjad.ahmad@polito.it \\ 3 Department of Applied Science and Technology, Politecnico di Torino, 10129 Turin, Italy; \\ jeanmarc.tulliani@polito.it \\ * Correspondence: qareebullah.ms14@nice.nust.edu.pk or qareebanwari@gmail.com \\ + Presented at the 1st International Conference on Smart Materials for Sustainable Construction-SMASCO \\ 2019, Luleå, Sweden, 10-12 December 2019.
}

Published: 18 November 2019

\begin{abstract}
An efficient and promising approach from economy point of view to effectively disperse Multi-walled carbon nanotubes (MWCNTs) in cementitious environment has been devised. The natural organic extract from species of indigenously known 'keekar' trees scattered along tropical and sub-tropical countries; is found as an exceptional replacement to the non-natural commercial surfactant. In the initial phase ideal content of surfactant was explored in relation with the added content of MWCNTs using ultra-violet spectroscopy. The experimental investigations were then extended to five formulations containing $0.0,0.025,0.05,0.08$ and 0.10 MWCNTs by weight percent of cement. It was observed that the natural surfactant produced efficient dispersion at much reduced cost (>14\% approx.) compared with the commercial alternate. The estimated weight efficiency factor ' $\varphi$ ' was found 6.5 times higher by the proposed sustainable replacement to the conventional with remarkable increase of $23 \%$ in modulus of rupture on $0.08 \mathrm{wt} \%$ addition of MWCNTs. Besides, strength enhancement the dispersed MWCNTs also improved the first crack and ultimate fracture toughness by $51.5 \%$ and $35.9 \%$, respectively. The field emission scanning electron microscopy of the cryofractured samples revealed efficient dispersion of MWCNTs in the matrix leading to the phenomenon of effective crack bridging and crack branching in the composite matrix. Furthermore, the proposed scheme significantly reduced the early age volumetric shrinkage by $39 \%$ to mitigate early age micro-cracks encouraging long lasting deteriorations from durability prospects.
\end{abstract}

Keywords: MWCNTS; Acacia Nilotica Gum; Triton X-100; Fracture; Crack Bridging; Cement Mortar; Early age Micro-Cracking; Sustainability

(C) 2019 by the authors. Licensee MDPI, Basel, Switzerland. This article is an open access article distributed under the terms and conditions of the Creative Commons Attribution (CC BY) license (http://creativecommons.org/licenses/by/4.0/). 